\title{
GRP78 silencing enhances hyperoxia-induced alveolar epithelial cell apoptosis via CHOP pathway
}

\author{
HONG-YAN LU ${ }^{1}$, XIAO-QING CHEN ${ }^{2}$, WEI TANG ${ }^{1}$, QIU-XIA WANG ${ }^{1}$ and JIE ZHANG ${ }^{1}$ \\ ${ }^{1}$ Department of Pediatrics, The Affiliated Hospital of Jiangsu University, Zhenjiang, Jiangsu 212001; \\ ${ }^{2}$ Department of Pediatrics, The First Affiliated Hospital of Nanjing Medical University, Nanjing, Jiangsu 210029, P.R. China
}

Received October 16, 2016; Accepted May 26, 2017

DOI: $10.3892 / \mathrm{mmr} .2017 .6681$

\begin{abstract}
Hyperoxia is one of the primary causes of bronchopulmonary dysplasia, which may occur in premature infants following supplemental oxygen therapy. Glucose regulated protein 78 (GRP78), which is a molecular chaperone located in the lumen of the endoplasmic reticulum (ER), has been reported to regulate hyperoxia-associated ER stress. The role of GRP78 in lung epithelial cells during hyperoxia remains to be elucidated. In the present study, the A549 cultured human lung epithelial cell line was exposed to hyperoxic conditions, and then transfected with short interfering (si)RNA targeted to GRP78. siRNA or pEGFP-N1 plasmid were used to knockdown or overexpress specific genes, reverse transcription-quantitative polymerase chain reaction and western blot analysis were used to detect RNA and protein levels of gene expression, and flow cytometry was used to detect apoptosis. The expression levels of ER stress-associated genes were determined, and a significant increase in C/EBP homologous protein (CHOP) expression and apoptosis of A549 cells was observed, following GRP78 knockdown. The overexpression of CHOP downregulated B-cell lymphoma (Bcl)-2 expression levels, upregulated BCL2 associated X (Bax), and increased apoptosis of A549 cells under conditions of hyperoxia. CHOP knockdown demonstrated the opposite effect on Bcl-2 and Bax expression levels. These results suggested that GRP78 silencing promoted lung epithelial cell apoptosis during hyperoxia, via regulation of the $\mathrm{CHOP}$ pathway.
\end{abstract}

\section{Introduction}

Bronchopulmonary dysplasia (BPD) is one of the most common chronic lung diseases in preterm infants undergo

Correspondence to: Dr Hong-Yan Lu, Department of Pediatrics, The Affiliated Hospital of Jiangsu University, 438 Jiefang Road, Zhenjiang, Jiangsu 212001, P.R. China

E-mail: hylu@ujs.edu.cn

Key words: A549, CHOP, glucose regulated protein 78, hyperoxia, siRNA supplemental oxygen therapy in US (1). Oxygen toxicity or hyperoxia is one of the major risk factors in the development of bronchopulmonary dysplasia. Hyperoxia was reported to promote cell injury in alveolar endothelial and epithelial cells in vivo and in vitro $(2,3)$, leading to impaired gas exchange and increased epithelial apoptosis (4). Premature infants are more susceptible to hyperoxia induced epithelial damage due to their respiratory immaturity and deficiency of anti-oxidant enzyme activity $(5,6)$. The molecular mechanism in regulating epithelial apoptosis under hyperoxia needs to be further elucidated.

The essential site responsible for protein synthesis and maturation is endoplasmic reticulum (ER), into which newly synthesized polypeptide chains enter through a peptide translocon, and undergo maturation processes such as cleavage, glycosylation, disulfide bond formation, folding and assembly. Much physiological and pathological stimulation, such as ischemia, hyperoxia, and poisons, cause ER stress, during which inhibition of protein glycosylation or disulfide bond formation results in accumulation of unfolded and misfolded proteins in the lumen of the ER. Gene expression alteration occurs during ER stress (7). Glucose regulated protein 78 (GRP78), a molecular chaperone, locates in the lumen of the ER that binds newly synthesized proteins as they translocate into the ER, and maintains them in a state competent for subsequent folding and oligomerization. GRP78 protein is usually highly induced by the microenvironment factors including hyperoxia, acidosis as well as glucose deprivation (8). Inositol-requiring enzyme-1 (IRE1), activating transcription factor-6 (ATF6), and protein kinase regulated by RNA-like ER kinase (PERK) play important role during ER stress (9). GRP78 recruitment to chaperone the malfolded proteins results in GRP78 dissociation from its conformational binding state of the above three trans-membrane receptors $(10,11)$, subsequently induces cell apoptosis. C/EBP homologous protein (CHOP) is widely known as a participator in the initiation of apoptosis. IRE1, ATF6 and PERK can trigger CHOP (12). The Bcl 2 family and plays a crucial role in the apoptotic process of various cancers. It has been documented that Bax (bcl-2-like protein 4) and Bcl-2 (B-cell lymphoma 2) are separately pro-apoptosis and anti-apoptosis proteins of the $\mathrm{Bcl} 2$ family and that these two molecules can finally regulate programmed cell death in ER (13). The pathway (via PERK) that induces transcription of the pro-apoptotic factor $\mathrm{CHOP}$ can inhibit anti-apoptotic 
protein Bcl-2, leading to activation of the executioner caspase-3 and cell death (14). mRNA and protein levels of GRP78 and CHOP were increased in lung tissue of preterm Sprague-Dawley rats exposed to hyperoxia (15). Exposure to $95-100 \% \mathrm{O}_{2}$ induces CHOP mRNA expression in the bronchiolar epithelium of adult mice and in isolated type II cells in culture (16), as well as postnatal day 2 and 7 murine developing lung tissue (17). Hyperoxia has also been shown to enhance CHOP expression after $72 \mathrm{~h}$ exposure together with increased ATF4 mRNA expression (18). However, how GRP78 regulates lung epithelial cell apoptosis during hyperoxia, especially the underlying mechanisms still remains unknown.

In this study, we used siRNA targeted GPR78 to transfect A549 cell under hyperoxia. GPR78 knockdown increased the expression of CHOP at gene level or protein level, further induced A549 cell apoptosis under hyperoxia. CHOP overexpression under hyperoxia led to the robust apoptosis of A549 cells by inducing Bax and inhibiting Bcl-2. CHOP knockdown (CHOP-siRNA) showed opposite effect on Bax and Bcl-2. GRP78 silencing promoted lung epithelial cells apoptosis during hyperoxia, probably through regulating CHOP pathway.

\section{Materials and methods}

Cell culture and hyperoxia exposure. The human airway epithelial cell line A549 was purchased from American Type Culture Collection (ATCC; Manassas, VA, USA) and cultured in DMEM/F12 with $10 \%$ FBS. Cells were grown under humidified conditions consisting of $95 \%$ air and $5 \% \mathrm{CO}_{2}$ at $37^{\circ} \mathrm{C}$ (normoxia). For hyperoxia exposure, cells were plated in an MIC-101 chamber (Modular Incubator; Billups-Rothenberg, Inc., Del Mar, CA, USA) filled with $95 \% \mathrm{O}_{2}$ and $5 \% \mathrm{CO}_{2}$ for up to $72 \mathrm{~h}$ at $37^{\circ} \mathrm{C}$. The gases were replaced every day.

Lipsome mediated cell transfection and hyperoxia treatment of A549. siRNA sequences targeted GRP78,CHOP were designed and called GRP78-siRNA and CHOP-siRNA, respectively. The sequences of GRP78-siRNA or CHOP-siRNA were as follows: 5'-AAGAUCACAAUCACCAAUGACTT-3', 5'-AAG AACCAGCAGAGGUCACAATT-3'. The sequence of the corresponding negative control was 5'-AAAUCAUAGCGU AUGGUGCUGTT-3'. 3e5 A549 cells cultured in six-well plate were transfected with $4 \mu \mathrm{g}$ of siRNA or pEGFP-N1 plasmid with CHOP by Lipofectamine ${ }^{\circledR} 2000$ (Invitrogen Life Technologies, Carlsbad, CA, USA). 24 h after transfection, fresh medium was added. Next, A549 cells were treated with hyperoxia for 24, 48 and 72 h subsequently. A549 cells without transefection or hyperoxia were designated as control (C). A549 cells treated with the combination of negative control transfection and hyperoxia were designated as negative control $(\mathrm{N})$.

Reverse transcription-quantitative polymerase chain reaction (RT-qPCR). RT-qPCR was carried out as previous described (15) to detect the expression of genes including ATF6, PERK, CHOP, Bcl-2 and Bax. Total RNA was extracted with Trizol ( $\sim 0.5 \mathrm{e} 6$ cells adding $0.5 \mathrm{ml}$ TRIzol) reagent (Applied Biosystems Life Technologies, Foster
City, CA, USA) and fractionated by electrophoresis on a $1.2 \%$ agarose/3-(N-morpholino) propanesulfonic acid/formaldehyde gel to ensure RNA integrity. Total RNA was reverse-transcribed to cDNA according to the manufacturer's instructions (Multiscribe Reverse Transcriptase; Applied Biosystems Life Technologies). Platinum Taq polymerase (Invitrogen Life Technologies) and EvaGreen dye (Biotium, Inc., Hayward, CA, USA) were applied for RT-qPCR. In this system, the increase in the concentration of EvaGreen dye fluorescent is proportional to the increase in PCR products; the reaction production can be accurately measured in the exponential phase of amplification by the ABI prism 7700 Sequence Detection System. The sequences of the primers used are listed in Table I. The signal of the housekeeping gene GAPDH was used for normalization. The correct size of PCR product was confirmed by electrophoresis on a $2 \%$ agarose gel stained with ethidium bromide. Metling curve analysis was performed to assess the specificity of the amplified PCR products.

Western blotting. Total protein was isolated using RIPA lysis buffer (Biyuntian Biotechnology Co., Ltd., Shanghai, China), and protein concentrations were determined by the Bradford method. $80 \mu \mathrm{g}$ proteins were separated by SDS-PAGE and transferred onto polyvinylidene fluoride membranes with the Bio-Rad Trans blot system. Membranes were stained by Ponceau for $3 \mathrm{~min}$, and cleaned by double distilled $\mathrm{H}_{2} \mathrm{O}$ until the red blots were clear. After blocking in 5\% bovine serum albumin (BSA) and mixture of Tris-Buffered Saline and Tween-20 (TBST) for $1 \mathrm{~h}$, membranes were incubated overnight in primary antibody diluted in $5 \% \mathrm{BSA}$ at $4^{\circ} \mathrm{C}$. The following primary antibodies were used: anti-PERK (AF5304), anti-IRE1 (DF7709), anti-ATF6 (DF6009), anti-CHOP (DF6025, 1:500, Affinity biosciences, USA), anti- $\beta$-actin (A1978, 1:10,000; Sigma, St. Louis, MO, USA). Membranes were incubated for $1 \mathrm{~h}$ with HRP-conjugated goat anti-mouse $(1: 5,000)$ or rabbit $(1: 2,000)$ immunoglobulin (Sigma) in $5 \%$ BSA. Chemiluminescence kit (Biyuntian Biotechnology Co., Ltd.) was used to visualize the secondary antibody. The bands were quantified using Image $\mathbf{J}$ software from three independent experiments.

Flow cytometry. Transfected cells were harvested, washed by ice-cold PBS, centrifuged at $300 \mathrm{x}$ g for $5 \mathrm{~min}$. $1 \mathrm{e} 6$ Cells were re-suspended with $500 \mu 1$ of 1 xbinding buffer, then stained with FITC-conjugated Annexin V and PI (Biyuntian Biotechnology Co., Ltd.) for $15 \mathrm{~min}$ at $4^{\circ} \mathrm{C}$ in the dark. The samples were analyzed using FACS (BD Biosciences, San Diego, CA, USA).

Statistical analysis. The results are expressed as the mean \pm standard error of the mean (SEM) of at least three independent experiments. All data were analyzed by SPSS 20.0 statistical software (SPSS, Inc., Chicago, IL, USA). Statistical analysis comparing the treated and control groups was assessed using the Student's t-test, and among multiple groups were tested by analysis of variance (ANOVA) followed by Geisser-Greenhouse corrections post hoc test. $\mathrm{P}<0.05$ was considered to indicate a statistically significant difference. 
Table I. Sequences of primers used in qPCR.

\begin{tabular}{lll}
\hline Gene & \multicolumn{1}{c}{ Sense } & \multicolumn{1}{c}{ Antisense } \\
\hline GRP78 & TCCTATGTCGCCTTCACT & ACAGACGGGTCATTCCAC \\
PERK & TTGTCGCCAATGGGATAG & CAGTCAGCAACCGAAACC \\
IRE1 & GACAGGCTCAATCAAATGG & CGGTCAGGAGGTCAATAACA \\
ATF6 & TCAATGGGCAGGACTACGA & GGGAGCCAAAGAAGGTGT \\
CHOP & CACTCTTGACCCTGCTTC & AGTCGCCTCTACTTCCCT \\
Bcl-2 & TCCAATCCTGTGCTGCTA & ACTCTGTGAATCCCGTTT \\
Bax & TTTTGCTTCAGGGTTTCATC & GACACTCGCTCAGCTTCTTG \\
GAPDH & GCACCGTCAAGGCTGAGAAC & TGGTGAAGACGCCAGTGGA
\end{tabular}

qPCR, quantitative polymerase chain reaction.

\section{Results}

GRP78 silencing increase CHOP expression in A549 cells under hyperoxia. We used GPR78-siRNA to transfect A549 cells, hyperoxia was established subsequently for 24,48 and $72 \mathrm{~h}$ after transfection, expressions of PERK, ATF6, IRE1 and $\mathrm{CHOP}$ at gene level and protein level were detected. GRP78 mRNA level dropped $80-90 \%$ by RT-PCR and protein level dropped over $50 \%$ by western blotting after knockdown (data not shown). The results showed that the expressions of PERK, ATF6 and IRE1 were not affected at gene level and protein level, while the expression of CHOP slightly increased after GPR78 silencing at gene level and protein level under normoxia, the increases were significantly enhanced under hyperoxia (Figs. 1 and 2). Also, the expression of CHOP in A549 cells treated with GRP78-siRNA and hyperoxia increased gradually with time. CHOP protein expression was slightly increased after $24 \mathrm{~h}$ under hypoxia after shamRNA treatment, not significantly different from those under nomaxia (Fig. 2E and F). Our previous studies have shown CHOP protein expression was increased for $\sim 2$ folds under hypoxia for $72 \mathrm{~h}$ (15), GRP78 knockdown under hypoxia for $72 \mathrm{~h}$ further brought up $\mathrm{CHOP}$ protein expression by $\sim 4.5$-folds.

The effect of GRP78-siRNA on the apoptosis of A549 cells under hyperoxia. A549 cells were treated with GRP78 siRNA and hyperoxia, induction of ER-mediated apoptosis was assessed. We found that that the under normal oxygen levels, the percent of apoptotic A549 cells was increased over time after GRP78 silencing, in addition, the apoptosis of A549 cells were further enhanced at presence of hyperoxia over time, which is consistent with the increase of CHOP expression after GRP79 silencing (Fig. 3).

The effect of $\mathrm{CHOP}$ overexpression on the expressions of $\mathrm{Bcl}-2$ or Bax under hyperoxia. To test whether CHOP regulation by GRP78 was correlated with the subsequent apoptotic events of A549 cells, we overexpressed CHOP by using pEGFP-N1-CHOP (plasmid containing core domain sequence of CHOP) on A549 cells, and establish hyperoxia subsequently for 24 , 48 and $72 \mathrm{~h}$ after transfection, and monitored the expressions of anti-apoptotic protein $\mathrm{Bcl}-2$ and pro-apoptotic protein Bax at gene level and protein level. CHOP overexpression induced
A

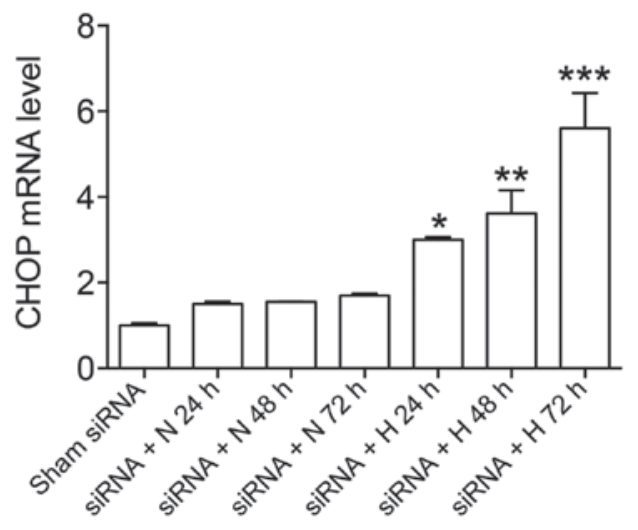

B

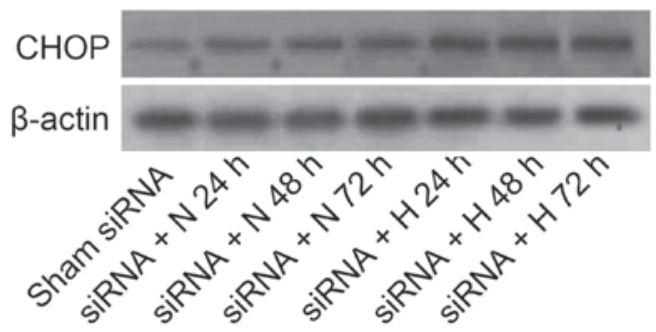

C

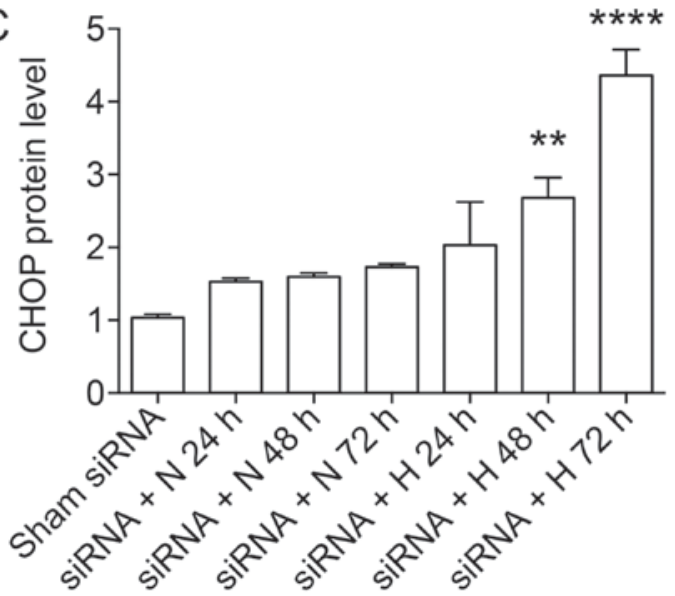

Figure 1. The expressions of CHOP detected by (A) RT-qPCR and (B and C) western blotting. A549 cells were transfected with GRP78-siRNA or negative control, hyperoxia was established subsequently for 24,48 and $72 \mathrm{~h}$ after transfection. Sham siRNA: A549 cells treated with negative control siRNA; siRNA+N 24 h, siRNA+N 48 h, siRNA+N 72 h: A549 cells were treated with GRP78-siRNA and nomaxia for 24, 48 and $72 \mathrm{~h}$; siRNA+H $24 \mathrm{~h}$, siRNA+H 48 h, siRNA+H 72 h: A549 cells were treated with GRP78-siRNA and hyperoxia for 24,48 and $72 \mathrm{~h}$. 

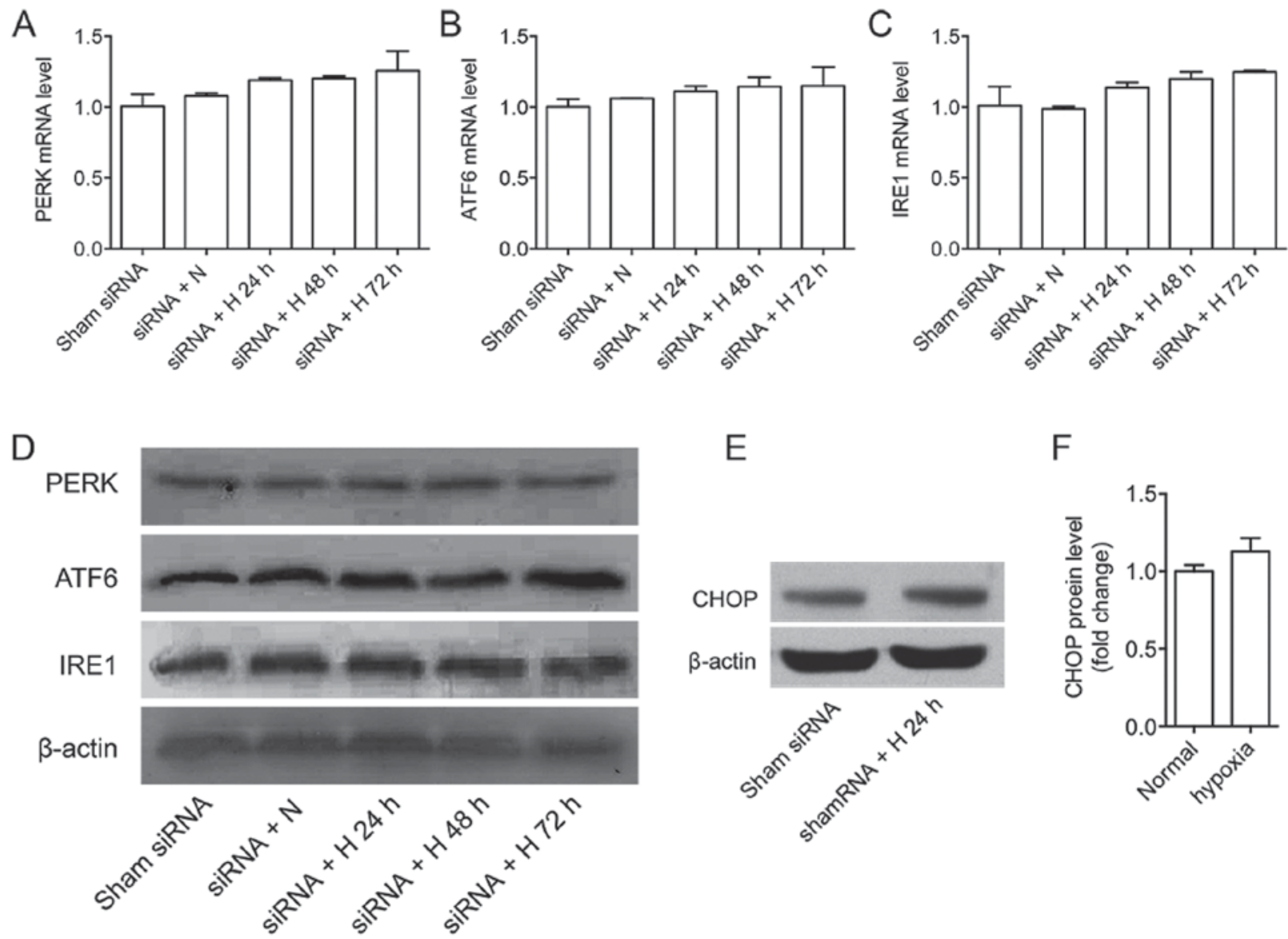

Figure 2. The expressions of PERK, ATF6, IRE1 were detected by (A-C) RT-qPCR and (D) western blotting. A549 cells were transfected with GRP78-siRNA or negative control, hyperoxia was established subsequently for 24,48 and $72 \mathrm{~h}$ after transfection. Sham siRNA: A549 cells treated with negative control siRNA; siRNA+N: A549 cells were treated with GRP78-siRNA and nomaxia for $24 \mathrm{~h}$; siRNA+H $24 \mathrm{~h}$, siRNA+H $48 \mathrm{~h}$, siRNA+H $72 \mathrm{~h}$ : A549 cells were treated with GRP78-siRNA and hyperoxia for 24, 48 and $72 \mathrm{~h}$. CHOP protein expression was slightly increased after $24 \mathrm{~h}$ under hypoxia after (E and F) shamRNA treatment.

2-3-folds mRNA ( $24 \mathrm{~h})$ by RP-PCR and protein expression levels $(48 \mathrm{~h}$ ) by western blot (data not shown). We found that the expression of Bcl-2 and Bax at RNA level were not affected by CHOP overexpression under normal oxygen level for up to $72 \mathrm{~h}$ after transfection. Decreased expression of Bcl-2 and increased expression of Bax at protein level were found in later time points after CHOP overexpression (48 and $72 \mathrm{~h}$ ). Importantly, CHOP overexpression under hyperoxia significantly decreased the expression of $\mathrm{Bcl}-2$ and increased expression of Bax at both RNA and protein level (Fig. 4). Bcl-2 and Bax protein levels were not changed after $24 \mathrm{~h}$ under hyperoxia compared with normoxia on empty vector treated cells (Fig. 5).

CHOP overexpression promoted apoptosis of A549 cells under hyperoxia. To examine whether regulation of Bcl-2 and Bax by CHOP could impact the apoptosis of A549 cells, we stained A549 cells with PI and Annexin V-FITC under normoxia or hyperoxia after CHOP overexpression. The percent of apoptotic A549 cells (defined by Annexin- $\mathrm{V}^{+} \mathrm{PI} \mathrm{I}^{-}$) increased over time after $\mathrm{CHOP}$ overexpression under normoxia using BD FACSCanto, furthermore, the apoptosis of A549 cells was significantly enhanced under hyperoxia, with the peak apoptotic phase at $48 \mathrm{~h}$ after hyperoxia treatment, suggesting the early apoptosis of A549 cells treated with pEGFP-N1-CHOP transfection under hyperoxia (Fig. 6).
The effect of CHOP-siRNA on the expression of Bcl-2 and Bax on A549 cells under hyperoxia. To further confirm the role of $\mathrm{CHOP}$ expression on regulation of apoptotic related genes, we used CHOP-siRNA to transfect A549 cell, established hyperoxia subsequently for 24,48 and $72 \mathrm{~h}$ after transfection, and monitored the expressions of $\mathrm{Bcl}-2$ and Bax at gene level and protein level. CHOP siRNA induced over $90 \%$ mRNA by RT-PCR and around $50-60 \%$ protein downregulation by western blot compared with shamRNA (data not shown). We found the relative mRNA expression of Bcl-2 was increased and Bax was decreased at later time points after CHOP siRNA at gene level and protein level, while significant increase of Bcl-2 and decrease of Bax were shown when A549 cells were treated with CHOP siRNA under hyperoxia. These results further confirmed the important role of CHOP and hyperoxia in promoting apoptosis of A549 cells, probably through regulation of $\mathrm{Bcl}-2$ and $\mathrm{Bax}$ (Fig. 7). Bcl-2 and Bax protein levels were not significantly changed after $24 \mathrm{~h}$ under hyperoxia compared to normoxia on Sham siRNA treated cells (Fig. 8).

\section{Discussion}

Hyperoxia-induced lung injury after oxygen supplementation is one of the major risk factors in the pathogenesis of BPD (1). 
A

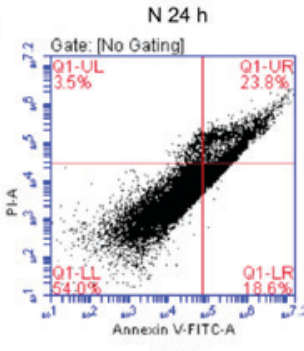

$\mathrm{H} 24 \mathrm{~h}$
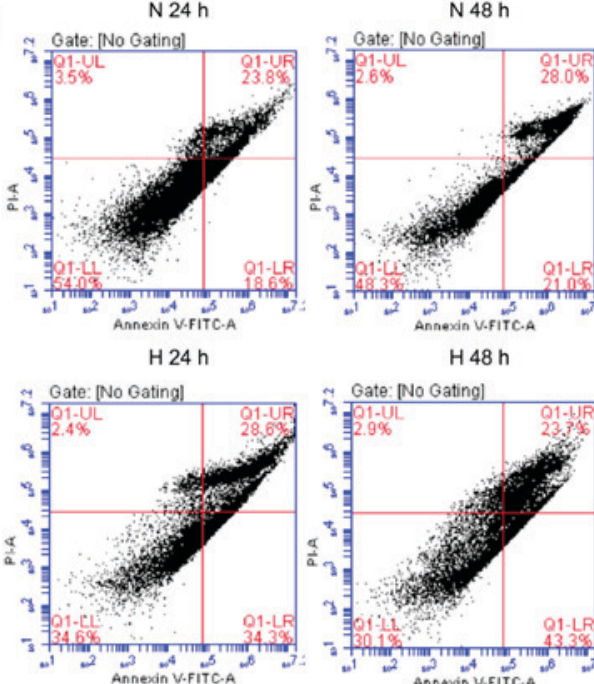

$\mathrm{H} 48 \mathrm{~h}$

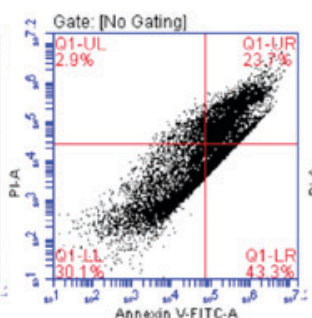

B

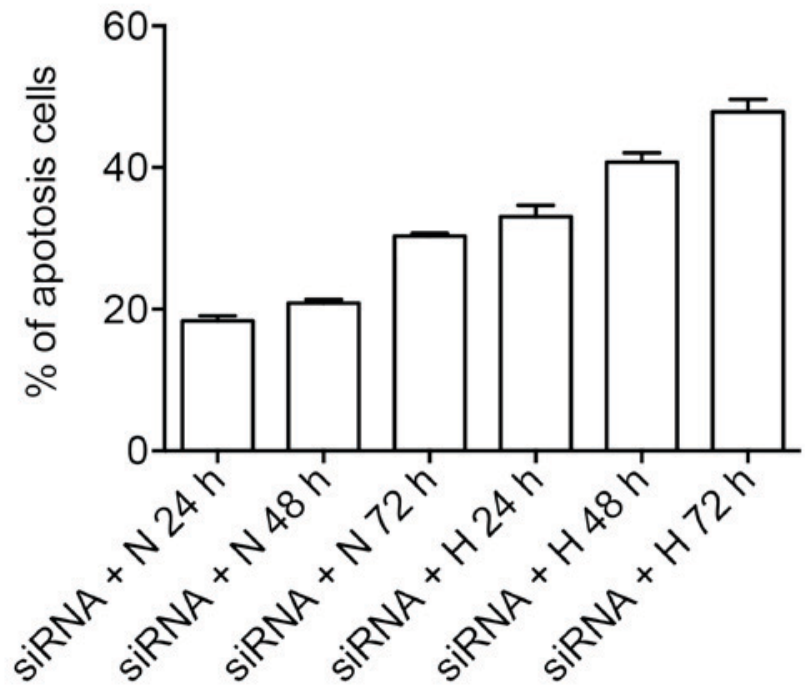

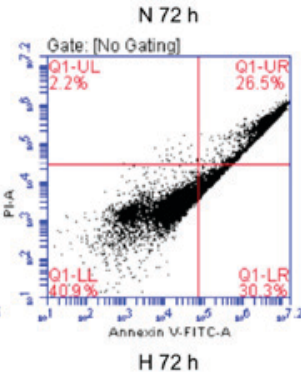

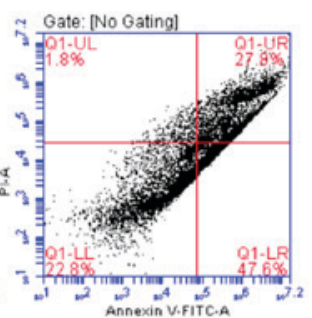

Figure 3. The apoptosis of A549 cells treated with GRP78-siRNA transfection under hyperoxia. (A) A549 cells were transfected with GRP78-siRNA, hyperoxia was established subsequently for 24, 48 and 72 h after transfection. Apoptosis was determined by flow cytometry. (B) N 24 h, N 48 h, N 72 h: A549 cells were treated with GRP78-siRNA and nomaxia for 24, 48 and 72 h; H 24 h, H 48 h, H 72 h: A549 cells were treated with GRP78-siRNA and hyperoxia for 24, 48 and $72 \mathrm{~h}$. Experiments were repeated three times and percentages of apoptotic cells were quantified.

Observations from prenatal and postnatal lung studies revealed that apoptosis plays an important role in lung development in animals as well as in humans. Apoptosis is more prominent in mesenchymal cells and less frequent in epithelium in developing lungs, which are normal processes in alveolar wall thinning and alveolar formation $(19,20)$. Preterm infants underwent supplemental oxygen therapy showed disrupted lung development featured by larger and simplified alveoli, increased alveolar macrophages, and thickened alveolar walls due to interstitial fibrosis and smooth muscle hyperplasia $(21,22)$. Hyperoxia causes apoptosis in peripheral airways $(23,24)$. It has demonstrated that apoptosis is significantly increased in alveolar epithelial cells in preterm infants with BPD and respiratory distress syndrome $(25,26)$. All these results suggest that adaptive apoptosis is a critical process in lung development, neonatal lung injury and the pathogenesis of BPD. In this study, the A549 cell line was selected for this study due to its human alveolar type II epithelial cell origin, we cultured A549 cells under $95 \% \mathrm{O}_{2}$ to mimics hyperoxia in vitro, apoptosis were induced and cellular events associated with apoptosis were evaluated. There are potential limitations of using A549 for this study, hTERT immortalized cell line, primary cultured cells, or a panel of cancer cell lines will be included in future study to confirm the findings we discovered on A549 cells.

Prolonged oxygen exposure regulates the expression of a variety of genes involved in cellular oxidative stress, cell cycle, growth, and death (27). GRP78 is a HSP70 molecular chaperone located in the lumen of the ER that binds newly synthesized proteins as they are translocated into the ER, and maintain them in a state competent for subsequent folding and oligomerization. Inhibition or downregulation of GRP78 has been demonstrated to increase ER stress-induced cell death in melanoma and cancer cells $(28,29)$. GRP78 siRNA lipoplex inhibited the growth of the renal carcinoma cell line, which highly expresses GRP78 basally (11). Previous studies showed that 2-deoxyglucose (2-dG), tunicamycin (TM), and cigarette smoke extract (CSE) treatments induced apoptosis of alveolar epithelial cells, downregulation of GRP78 expression by GRP78 siRNA led to the increased expression of caspase-3 and sensitivity to apoptosis $(30,31)$. ER protein ERp57 
A

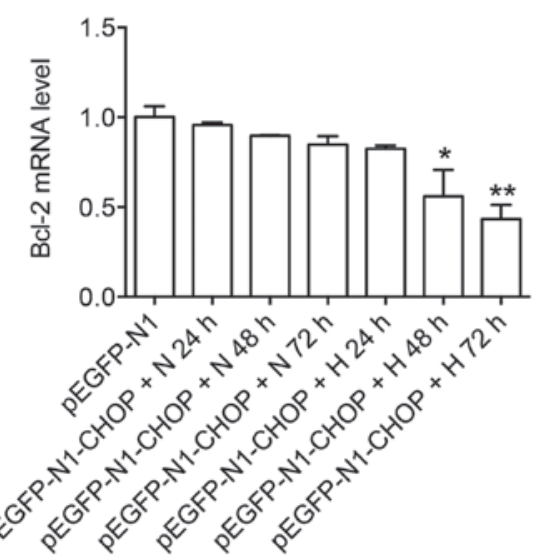

B

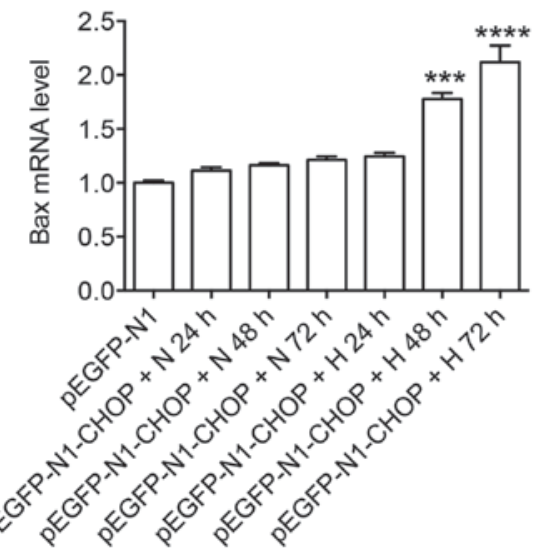

C Bcl-2

Bax

$\beta$-actin

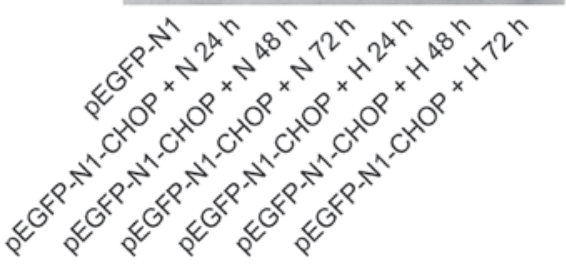

D
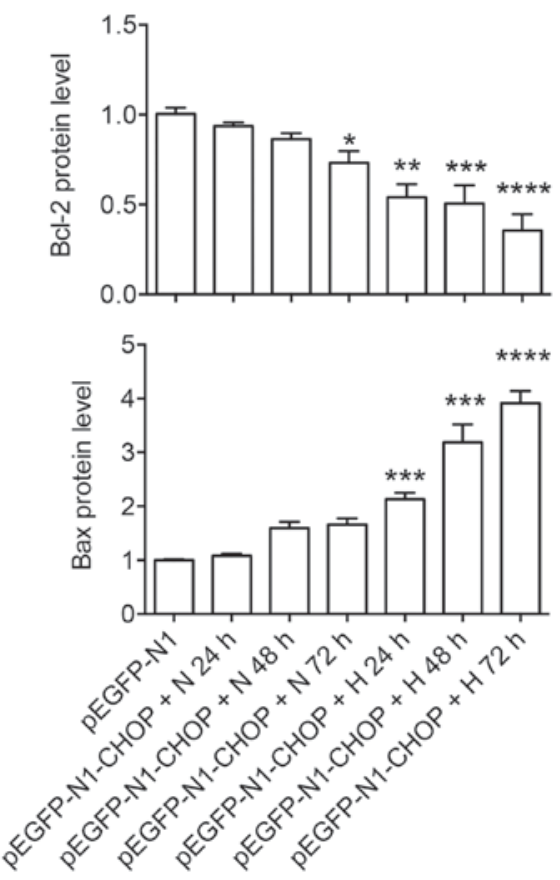

Figure 4. The expressions of Bcl-2 and Bax detected by (A and B) RT-qPCR and (C) western blotting. A549 cells were transfected with pEGFP-N1-CHOP or empty pEGFP-N1 vector (pEGFP-N1), hyperoxia was established subsequently for 24, 48 and $72 \mathrm{~h}$ after transfection. pEGFP-N1-CHOP-N24 h, pEGFP-N1-CHOP-N48 $\mathrm{h}$ and pEGFP-N1-CHOP-N72h: A549 cells were treated with pEGFP-N1-CHOP and nomaxia for 24,48 and $72 \mathrm{~h}$; pEGFP-N1-CHOP-H24 h, pEGFP-N1-CHOP-H48 h and pEGFP-N1-CHOP-H72h: A549 cells were treated with pEGFP-N1-CHOP and hyperoxia for 24, 48, and $72 \mathrm{~h}$. Experiments were repeated three times and band intensity of (D) Bcl-2 and Bax were quantified.

A

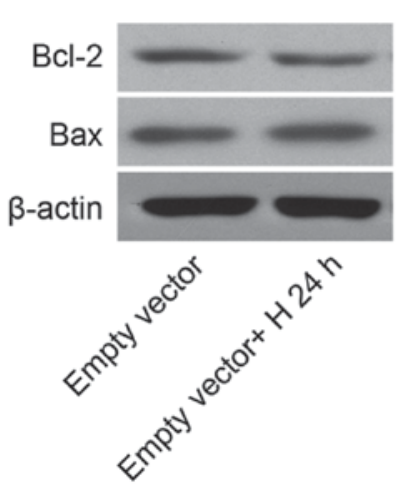

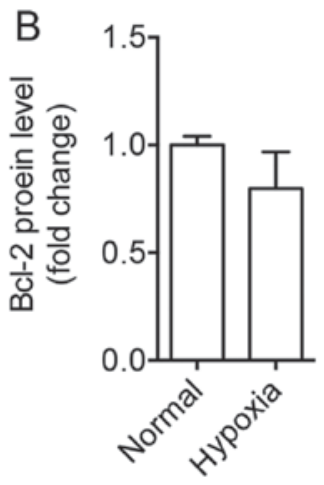

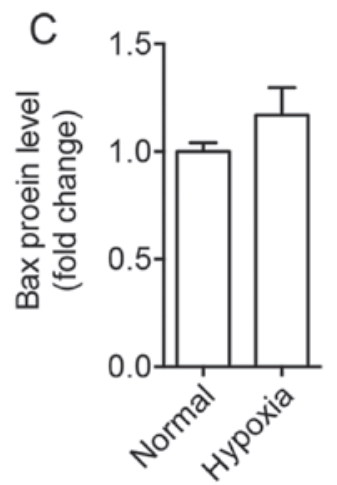

Figure 5. The expressions of Bcl-2 and Bax detected by (A) western blotting and (B and C) quantified. A549 cells were transfected with empty pEGFP-N1 vector (pEGFP-N1), hyperoxia was established subsequently for $24 \mathrm{~h}$.

knockdown protected hyperoxia- or tunicamycin-induced apoptosis of A549 cells by induction of BiP/GRP78 (8). In current study, we observed significant increase of apoptosis of A549 cells treated with the combination of GRP78-siRNA 
A
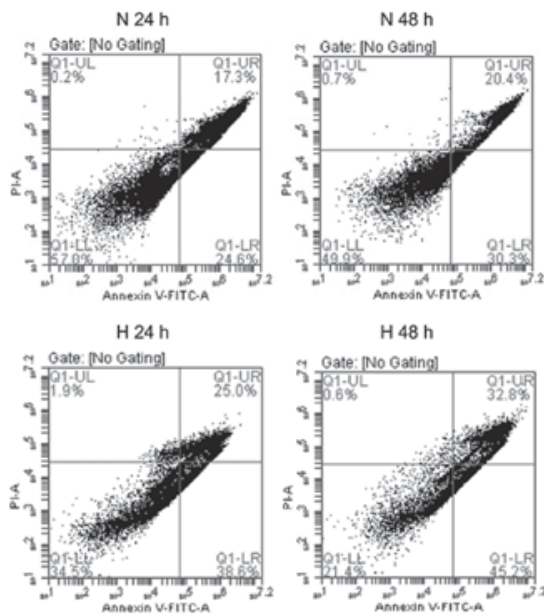

$\mathrm{H} 48 \mathrm{~h}$

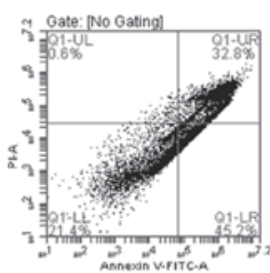

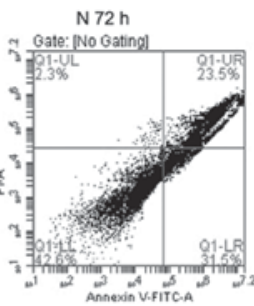

$\mathrm{H} 72 \mathrm{~h}$

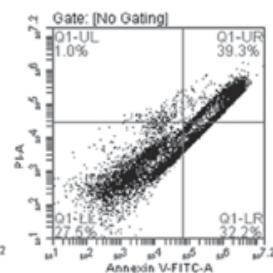

B
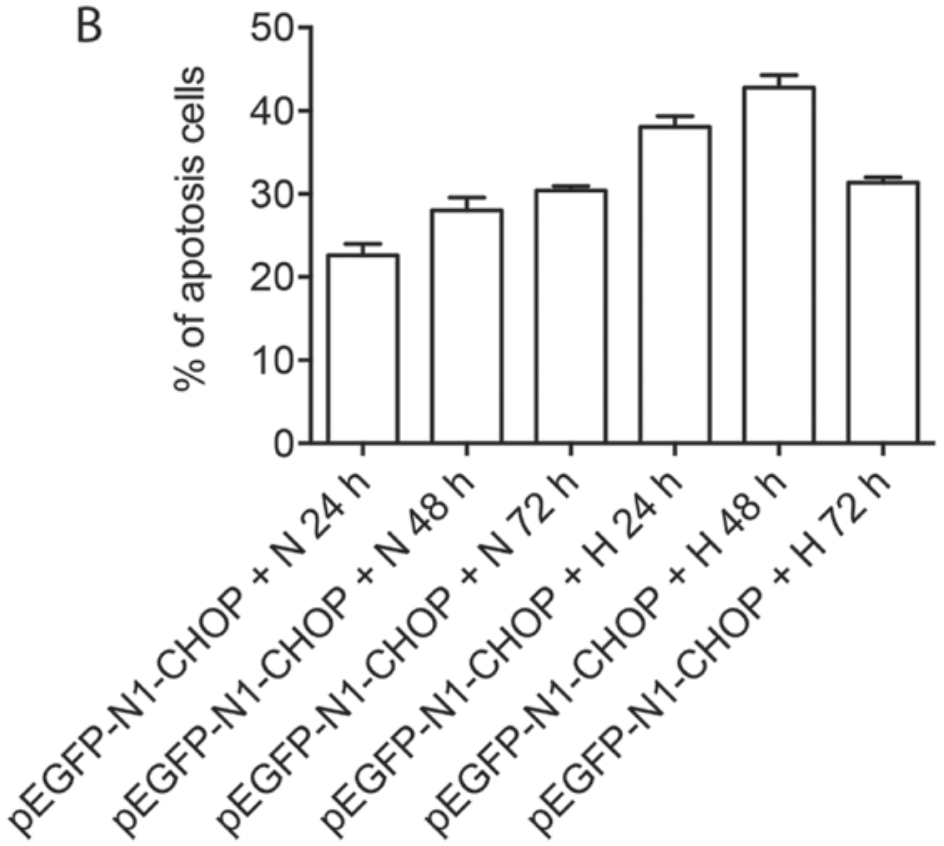

Figure 6. The apoptosis of A549 cells treated with pEGFP-N1-CHOP transfection under hyperoxia. A549 cells were transfected with pEGFP-N1-CHOP or empty pEGFP-N1 vector (pEGFP-N1), hyperoxia was established subsequently for 24,48 and 72 h after transfection. pEGFP-N1-CHOP-N24 h, pEGFP-N1-CHOP-N48 h and pEGFP-N1-CHOP-N72h: A549 cells were treated with pEGFP-N1-CHOP and nomaxia for 24, 48 and 72 h; pEGFP-N1-CHOP-H24 h, pEGFP-N1-CHOP-H48h and pEGFP-N1-CHOP-H72h: A549 cells were treated with pEGFP-N1-CHOP and hyperoxia for 24, 48 and $72 \mathrm{~h}$. The apoptotic cells were detected by flow cytometry with (A) PI and Annexin V-FITC staining and (B) quantified.

under hyperoxia, suggesting that GRP78 signaling pathway plays an important role for lung epithelial injuries in preterm infants undergo supplemental oxygen therapy.

ER stress induced cell death signaling occur via three ER-resident transmembrane proteins, IRE-1 $\alpha$, ATF6 $\alpha$, and PERK (14,32): i) Activated IRE-1 $\alpha$ can recruit c-Jun N-terminal inhibitory kinase (JIK) and tumor necrosis factor receptor-associated factor-2 (TRAF2) to activate apoptosis-signal regulating kinase-1 and c-Jun N-terminal kinase (JNK), leading to the activation of a mitochondria-dependent cell-death pathway (activation of caspase-3, 8, 9, Bcl-2-associated $\mathrm{X}$ protein or Bax, the release of cytochrome $c$ ); ii) The release of JIK from procaspase-12 allows for activation to caspase-12, which activates procaspase-9, which in turn activates procaspase-3, the executioner of cell death. iii) Activated PERK phosphorylates the eukaryotic initiation factor- $2 \alpha$ that enhances the translation of ATF4 mRNA, which in turn induces CHOP $(33,34)$. CHOP can inhibit antiapoptotic Bcl-2, leading to the activation of the executioner caspase-3. Newborn murine lung exposed to hyperoxia and IFN- $\gamma$ showed marked increase in cyclooxygenase-2 (Cox2) and the upregulation of the endoplasmic reticulum (ER) stress pathway mediator $\mathrm{CHOP}$, which resulted in increased alveolar epithelial cell death in as well as murine BPD (17). We investigate the effects of GRP78 siRNA on the gene expression profile of ER stress signaling pathway molecules, and found that both gene and protein expression of $\mathrm{CHOP}$ increased compared with those treated with sham siRNA. Overexpression of CHOP under hyperoxia caused significant downregulation of Bcl-2 and upregulation of Bax, enhanced apoptosis of A549 cells, which suggested that imbalance of $\mathrm{CHOP}$ expression by GRP78 knockdown under hyperoxia might be the cause of increased apoptosis of A549 cells. Mouse embryo fibroblasts from $\mathrm{Bax}^{-/} \mathrm{Bak}^{-/}$mice were resistant to apoptosis induced by ER stress, suggesting the role of Bax and Bak as executioner 
A

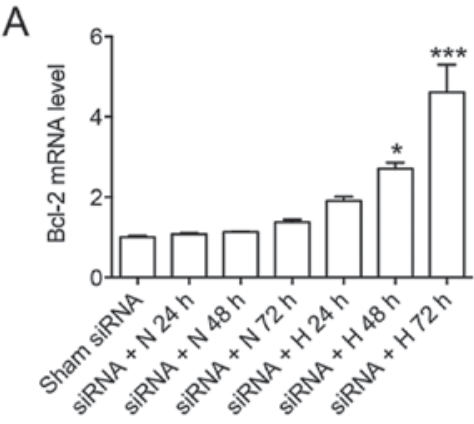

B

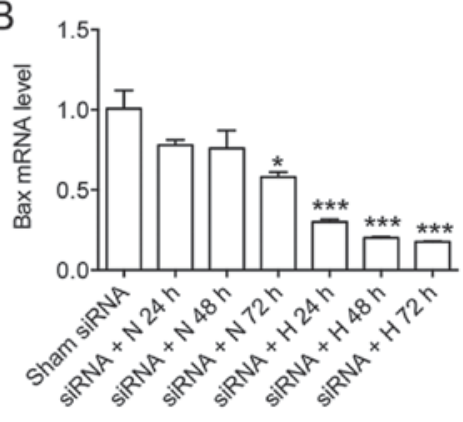

C BCl-2

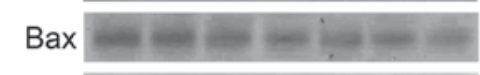

$\beta$-actin

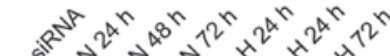

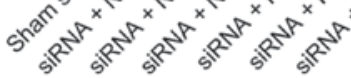
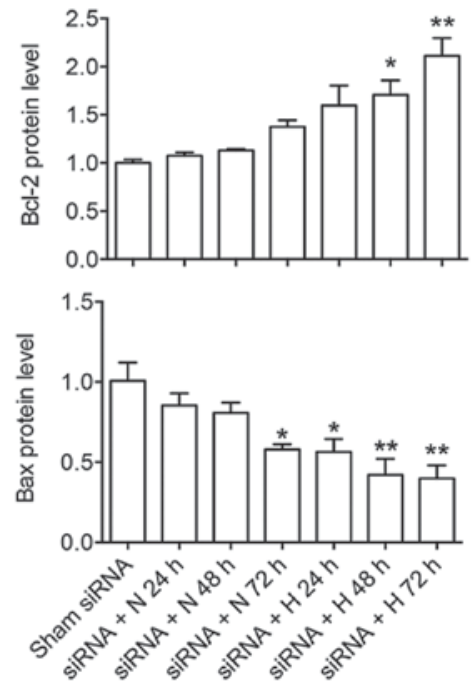

Figure 7. The expressions of Bcl-2 and Bax detected by qRT-PCR (A and B) and western blotting (C). A549 cells were transfected with CHOP-siRNA or sham control siRNA (Sham siRNA), hyperoxia was established subsequently for 24,48 and $72 \mathrm{~h}$ after transfection. siRNA+N24h, siRNA+N48h and siRNA+N72h: A549 cells were treated with CHOP siRNA and nomaxia for 24, 48 and $72 \mathrm{~h}$; siRNA+H24 h, siRNA+H48 h and siRNA+H72h: A549 cells were treated with CHOP siRNA and hyperoxia for 24, 48 and $72 \mathrm{~h}$. Experiments were repeated three times and band intensity of Bcl-2 and Bax were quantified (D).

A

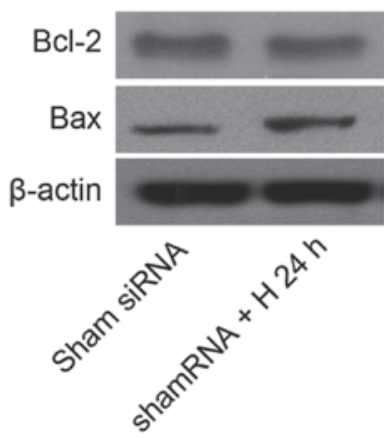

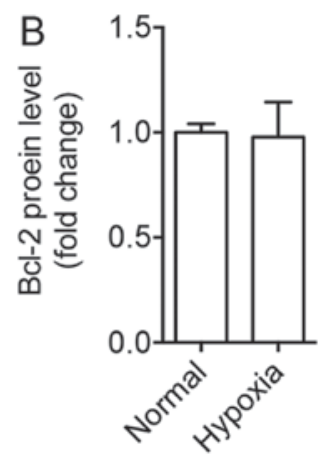

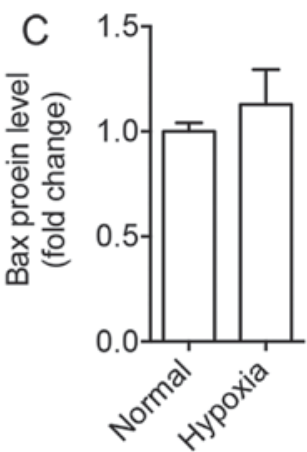

Figure 8. The expressions of Bcl-2 and Bax detected by western blotting (A) and quantified (B and C). A549 cells were transfected with Sham SiRNA, hyperoxia was established subsequently for $24 \mathrm{~h}$.

in ER-stress mediated apoptosis (35). Also, Matsumoto et al reported that overexpression of Bcl-2 blocked CHOP-induced apoptosis (36). On the other hand, we treated A549 cells with the CHOP siRNA under hyperoxia, the results showed the expression of Bcl-2 increased, while the expression of Bax decreased, which further supported our hypothesis on the role of GRP78-CHOP-Bcl-2/Bax pathway in ER related apoptosis of lung epithelial cells under hyperoxia.

In this study, we determined the effect of GRP78 siRNA and CHOP overexpression under hyperoxia on human lung epithelial cell line A549 cells, and found that the GRP78 siRNA or CHOP overexpression could lead the enhanced apoptosis of A549 cells under hyperoxia, suggesting that the important role of GRP78 in promoting lung epithelial apoptosis under hyperoxia, probably through regulating $\mathrm{CHOP}-\mathrm{Bcl}-2 / \mathrm{Bax}$ pathway, targeting GRP78 might help to reduce lung epithelial injury for preterm infants undergo oxygen supplementary treatment and lower the incidence of BPD.

\section{Acknowledgements}

This study was supported by the National Natural Science Foundation of China (NNSFC) (nos. 81370746 and no. 81300521), the Natural Science Foundation of Jiangsu Province, China (no. BK20161356), and the Social Development Foundation of Zhenjiang, China (no. SH2015071). 


\section{References}

1. Eichenwald EC and Stark AR: Management and outcomes of very low birth weight. N Engl J Med 358: 1700-1711, 2008

2. McGrath-Morrow SA and Stahl J: Apoptosis in neonatal murine lung exposed to hyperoxia. Am J Respir Cell Mol Biol 25 $150-155,2001$

3. De Paepe ME, Mao Q, Chao Y, Powell JL, Rubin LP and Sharma S: Hyperoxia-induced apoptosis and Fas/FasL expression in lung epithelial cells. Am J Physiol Lung Cell Mol Physiol 289: L647-L659, 2005.

4. Bourbon J, Boucherat O, Chailley-Heu B and Delacourt C: Control mechanisms of lung alveolar development and their disorders in bronchopulmonary dysplasia. Pediatr Res 57: 38R-46R, 2005

5. Frank L and Groseclose EE: Preparation for birth into an O2-rich environment: The antioxidant enzymes in the developing rabbit lung. Pediatr Res 18: 240-244, 1984.

6. Tanswell AK and Freeman BA: Pulmonary antioxidant enzyme maturation in the fetal and neonatal rat. I. Developmental profiles. Pediatr Res 18: 584-587, 1984.

7. Xu C, Bailly-Maitre B and Reed JC: Endoplasmic reticulum stress: Cell life and death decisions. J Clin Invest 115: 2656-2664, 2005.

8. Xu D, Perez RE, Rezaiekhaligh MH, Bourdi M and Truog WE: Knockdown of ERp57 increases BiP/GRP78 induction and protects against hyperoxia and tunicamycin-induced apoptosis. Am J Physiol Lung Cell Mol Physiol 297: L44-L51, 2009.

9. Maurel M and Chevet E: Endoplasmic reticulum stress signaling: The microRNA connection. Am J Physiol Cell Physiol 304: C1117-C1126, 2013.

10. Ozcan U, Cao Q, Yilmaz E, Lee AH, Iwakoshi NN, Ozdelen E, Tuncman G, Görgün C, Glimcher LH and Hotamisligil GS Endoplasmic reticulum stress links obesity, insulin action, and type 2 diabetes. Science 306: 457-461, 2004.

11. Wu J, Ruas JL, Estall JL, Rasbach KA, Choi JH, Ye L, Boström P, Tyra HM, Crawford RW, Campbell KP, et al: The unfolded protein response mediates adaptation to exercise in skeletal muscle through a PGC-1 $\alpha /$ ATF6 $\alpha$ complex. Cell Metab 13: 160-169, 2011.

12. Puthalakath H, O'Reilly LA, Gunn P, Lee L, Kelly PN, Huntington ND, Hughes PD, Michalak EM, McKimm-Breschkin J,Motoyama N, et al: ER stress triggers apoptosis by activating BH3-only protein Bim. Cell 129: 1337-1349, 2007.

13. Zinkel S, Gross A and Yang E: BCL2 family in DNA damage and cell cycle control. Cell Death Differ 13: 1351-1359, 2006.

14. Rutkowski DT and Kaufman RJ: That which does not kill me makes me stronger: Adapting to chronic ER stress. Trends Biochem Sci 32: 469-476, 2007.

15. Lu HY, Zhang J, Wang QX, Tang W and Zhang LJ: Activation of the endoplasmic reticulum stress pathway involving CHOP in the lungs of rats with hyperoxia-induced bronchopulmonary dysplasia. Mol Med Rep 12: 4494-4500, 2015.

16. O'Reilly MA, Staversky RJ, Watkins RH, Maniscalco WM and Keng PC: p53-independent induction of GADD45 and GADD153 in mouse lungs exposed to hyperoxia. Am J Physiol Lung Cell Mol Physiol 278: L552-L559, 2000.

17. Choo-Wing R, Syed MA, Harijith A, Bowen B, Pryhuber G, Janér C, Andersson S, Homer RJ and Bhandari V: Hyperoxia and interferon- $\gamma$-induced injury in developing lungs occur via cyclooxygenase- 2 and the endoplasmic reticulum stress-dependent pathway. Am J Respir Cell Mol Biol 48: 749-757, 2013.
18. Lozon TI, Eastman AJ, Matute-Bello G, Chen P, Hallstrand TS and Altemeier WA: PKR-dependent CHOP induction limits hyperoxia-induced lung injury. Am J Physiol Lung Cell Mol Physiol 300: L422-L429, 2011.

19. Dieperink HI, Blackwell TS and Prince LS: Hyperoxia and apoptosis in developing mouse lung mesenchyme. Pediatr Res 59: 185-190, 2006.

20. Bruce MC, Honaker CE and Cross RJ: Lung fibroblasts undergo apoptosis following alveolarization. Am J Respir Cell Mol Biol 20: 228-236, 1999.

21. Stenmark KR and Abman SH: Lung vascular development: Implications for the pathogenesis of bronchopulmonary dysplasia. Annu Rev Physiol 67: 623-661, 2005.

22. Thibeault DW, Mabry S and Rezaiekhaligh M: Neonatal pulmonary oxygen toxicity in the rat and lung changes with aging. Pediatr Pulmonol 9: 96-108, 1990.

23. Scavo LM, Ertsey R, Chapin CJ, Allen L and Kitterman JA: Apoptosis in the development of rat and human fetal lungs. Am J Respir Cell Mol Biol 18: 21-31, 1998

24. Kresch MJ, Christian C, Wu F and Hussain N: Ontogeny of apoptosis during lung development. Pediatr Res 43: 426-431, 1998.

25. Hargitai B, Szabó V, Hajdú J, Harmath A, Pataki M, Farid P, Papp Z and Szende B: Apoptosis in various organs of preterm infants: Histopathologic study of lung, kidney, liver, and brain of ventilated infants. Pediatr Res 50: 110-114, 2001.

26. May M, Strobel P, Preisshofen T, Seidenspinner S, Marx A and Speer CP: Apoptosis and proliferation in lungs of ventilated and oxygen-treated preterm infants. Eur Respir J 23: 113-121, 2004.

27. O'Reilly MA: DNA damage and cell cycle checkpoints in hyperoxic lung injury: Braking to facilitate repair. Am J Physiol Lung Cell Mol Physiol 281: L291-L305, 2001.

28. Stuhr LE, Raa A, Oyan AM, Kalland KH, Sakariassen PO, Petersen K, Bjerkvig R and Reed RK: Hyperoxia retards growth and induces apoptosis, changes in vascular density and gene expression in transplanted gliomas in nude rats. J Neurooncol 85 : 191-202, 2007.

29. Martin S, Hill DS, Paton JC, Paton AW, Birch-Machin MA, Lovat PE and Redfern CP: Targeting GRP78 to enhance melanoma cell death. Pigment Cell Melanoma Res 23: 675-682, 2010,

30. Ahmad M, Hahn IF and Chatterjee S: GRP78 up-regulation leads to hypersensitization to cisplatin in A549 lung cancer cells. Anticancer Res 34: 3493-3500, 2014.

31. He B, Luo B, Chen Q and Zhang L: Cigarette smoke extract induces the expression of GRP78 in A549 cells via the p38/MAPK pathway. Mol Med Rep 8: 1683-1688, 2013

32. Zhang K and Kaufman RJ: The unfolded protein response: A stress signaling pathway critical for health and disease. Neurology 66 (2 Suppl 1): S102-S109, 2006.

33. Liu G, Su L, Hao X, Zhong N, Zhong D, Singhal S and Liu X: Salermide up-regulates death receptor 5 expression through the ATF4-ATF3-CHOP axis and leads to apoptosis in human cancer cells. J Cell Mol Med 16: 1618-1628, 2012.

34. Konsavage WM, Zhang L, Wu Y and Shenberger JS : Hyperoxia-induced activation of the integrated stress response in the newborn rat lung. Am J Physiol Lung Cell Mol Physiol 302: L27-L35, 2012

35. Wei MC, Zong WX, Cheng EH, Lindsten T, Panoutsakopoulou V, Ross AJ, Roth KA, MacGregor GR, Thompson CB and Korsmeyer SJ: Proapoptotic BAX and BAK: A requisite gateway to mitochondrial dysfunction and death. Science 292: 727-730 2001.

36. Matsumura K, Sakai C, Kawakami S, Yamashita F and Hashida M: Inhibition of cancer cell growth by GRP78 siRNA lipoplex via activation of unfolded protein response. Biol Pharm Bull 37: 648-653, 2014. 